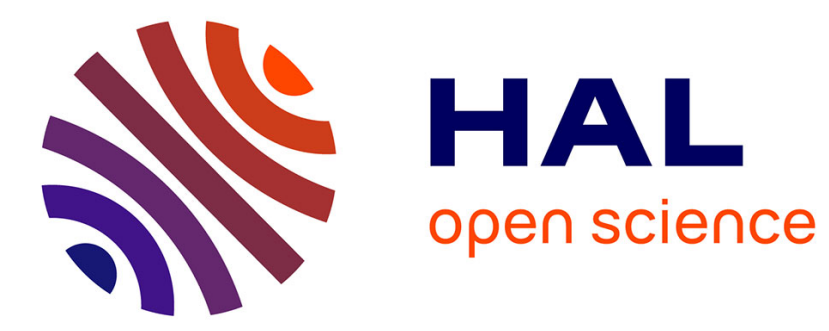

\title{
Un bon livre, Le Livre, les livres. L'Areopagitica de John Milton: de la liberté de la presse à la presse de la liberté
}

Christophe Tournu

\section{- To cite this version:}

Christophe Tournu. Un bon livre, Le Livre, les livres. L'Areopagitica de John Milton: de la liberté de la presse à la presse de la liberté. Laïcité. La pensée et les hommes, 1999, 48 (1), pp.41-54. hal-03135385

\section{HAL Id: hal-03135385 \\ https://hal.science/hal-03135385}

Submitted on 8 Feb 2021

HAL is a multi-disciplinary open access archive for the deposit and dissemination of scientific research documents, whether they are published or not. The documents may come from teaching and research institutions in France or abroad, or from public or private research centers.
L'archive ouverte pluridisciplinaire HAL, est destinée au dépôt et à la diffusion de documents scientifiques de niveau recherche, publiés ou non, émanant des établissements d'enseignement et de recherche français ou étrangers, des laboratoires publics ou privés. 


\section{Un bon livre, le Livre, les livres. L'Areopagitica de John Milton : de} la liberté de la presse à la presse de la liberté

\section{Christophe Tournu}

\section{Citer ce document / Cite this document :}

Tournu Christophe. Un bon livre, le Livre, les livres. L'Areopagitica de John Milton : de la liberté de la presse à la presse de la liberté. In: XVII-XVIII. Bulletin de la société d'études anglo-américaines des XVIle et XVIIle siècles. N48, 1999. pp. 41-54;

doi : https://doi.org/10.3406/xvii.1999.1453

https://www.persee.fr/doc/xvii_0291-3798_1999_num_48_1_1453

Fichier pdf généré le 27/03/2018 
UN BON LIVRE, LE LIVRE, LES LIVRES

L'AREOPAGITICA DE JOHN MILTON :

DE LA LibERTÉ de LA PRESSE

À LA PRESSE DE LA LIBERTÉ

Il en va des livres comme des hommes, écrit Milton. Certains seront adulés, d'autres rejetés - selon qu'ils auront bien ou mal agi. ${ }^{1}$ Sir Francis Bacon, dans An Advertisement Touching the Controversies of the Church of England, qu'il rédige en 1589 au plus fort de la crise des Marprelate Tracts, distinguait deux sortes de livres: d'une part, les écrits interdits: œuvre des non-conformistes, ils étaient dérangeants en ce qu'ils jetaient des étincelles de vérité à la face de l'Église établie; d'autre part, les écrits autorisés: œuvre du haut clergé, ils étaient temporis voces en ce qu'ils émanaient de l'orthodoxie. Selon l'humaniste, il fallait supprimer les deux sortes de livres parce qu'ils étaient également condamnables: "as the former sort doth deface the government of the church in the persons of the bishops and prelates, so the other doth lead into contempt the exercises of religion in the persons of sundry preachers." ${ }^{2} \mathrm{Si}$, pour Milton, la distinction est encore valable dans les années 1640 , il en arrive à des conclusions radicalement différentes.

Toutefois, comme ceux de Sir Francis, les écrits de Milton ne sont pas le produit de la raison pure, mais les idées exposées dans ses œuvres relèvent de circonstances particulières. En effet, Milton se destinait à la prêtrise anglicane: s'estimant bouté hors de l'Église par les Prélats (Reason

1. Cet article s'inspire en partie de ma thèse de doctorat, "Théologie et politique dans l'œuvre en prose de John Milton," U de Clermont-Ferrand II, juin 1996, 501 pp. Toutes les références se feront à John Milton, Complete Prose Works of John Milton, ed. Don Marion Wolfe, 8 vols. (New Haven: Yale UP, 1953-82).

2. James Spedding, ed., The Letters and the Life of Francis Bacon Including All His Occasional Works, 3 vols. (London: Longman, Green and Roberts, 1861) 1: 78. Voir "some have sought the truth in the conventicles and conciliables of heretics and sectaries, and others in the extern face and representation of the church; and both sorts have been seduced" (1: 74). Milton cite Bacon dans Areopagitica 2: 534, 542. 
of Church-Governement 1: 822-23), il leur lance cinq pamphlets antiépiscopaux. Sa jeune femme, Mary Powell, abandonne le domicile conjugal au bout d'un mois de mariage: et Milton de justifier le divorce pour incompatibilité d'humeur. On essaie de censurer la première édition de Doctrine and Discipline of Divorce (23 juin 1643): Milton se lance aussitôt dans un vibrant plaidoyer pour la liberté de la presse. Rétrospectivement dans sa Pro populo anglicano defensio (1651), il dira qu'il envisageait là, selon un plan prédéfini, le dernier aspect de la liberté personnelle. En fait, comme l'affirme Olivier Lutaud dans l'introduction à sa traduction de 1969, "sans [le] scandale [que suscita son pamphlet sur le divorce], il n'y aurait probablement pas eu Areopagitica." ${ }^{3}$ Les Presbytériens, ceux-là mêmes que Milton avait défendus dans $A n$ Apology against a Pamphlet, dénoncèrent violemment, avec Herbert Palmer, la parution illégale d'"un vilain livre" dédié au Parlement: "a wicked booke is abroad and uncensured, though deserving to be burnt, whose Author hath been so impudent as to set his Name to it, and dedicate it to your selves." ${ }^{4} \mathrm{La}$ défense du divorce était vue comme l'apologie du mariage adultère, "for Liberty to marry incestuously." Le 16 septembre 1644, William Prynne, dans Twelve Considerable Serious Questions, qualifiait le divorcisme de Milton, ainsi que la théorie de la mortalité de l'âme, que l'on attribue à Richard Overton, d"'Anabaptisticall, Antinomian, Hereticall, Atheisticall opinions." ${ }^{5}$ Daniel Featley, dans The Dippers Dipt, déplore: "the bonds of matrimony are let loose to inordinate lust." ${ }^{6}$ On essaya de faire interdire l'ouvrage de Milton sur la séparation de partenaires mal assortis: "ye have been instigated to a hard censure of [my] former book entitl'd, The Doctrine, and Discipline of Divorce" (Tetrachordon 2: 578).

Dans une protestation qu'elle adresse à la Chambre des Communes, le 24 août 1644, la Corporation des libraires se plaignait de la publication d'un ouvrage non autorisé, ni enregistré, de surcroît "scandaleux." Une enquête est ouverte. Milton devra comparaître devant les Lords au début de 1645: "The Lords . . . whether approving the doctrine, or not favoring

3. Milton, Areopagitica, 1644, ed. Olivier Lutaud (Paris: Aubier-Flammarion, 1969) 30 .

4. Herbert Palmer, The Glasse of Gods Providence towards His Faithfull Ones [sermon prononcé le 13 août, publié le 7 novembre 1644] 57.

5. William Prynne, Twelve Considerable Serious Questions (London, 16 September 1644) 7.

6. Daniel Featley, The Dippers Dipt (London, 7 February 1645) 57. 
his accusers, soon dismissed him." ${ }^{7}$ Milton est relaxé. Ce faisant, il n'est pas surprenant de voir Areopagitica s'en prendre à l'examen de la presse, exigé par le pouvoir avant qu'il n'en autorise la publication; Milton ne supporte pas qu'un livre doive se présenter devant un jury avant de naître au monde (2: 505-06). Ailleurs, dans sa Pro populo anglicano defensio secunda (1654), il déplore que le chercheur ne puisse publier le fruit de ses travaux s'il ne les a soumis à l'approbation d'une poignée d'individus prompts à condamner, envieux, bornés ou plein de préjugés (4: 679). Résultat: les Anglais seront réduits à ne plus rien connaître sinon ce que mesure le boisseau des censeurs; si l'écrit dépasse l'entendement du vulgaire, il ne verra pas le jour $(626,679) .{ }^{8}$ Cette vue, Milton l'exprime alors que, nommé Secrétaire aux Affaires étrangères de la République, il a lui-même exercé les fonctions de censeur. On dira, pour sa défense, que son examen du Mercurius politicus ne pouvait être que de principe, dans la mesure où il s'agissait là d'une publication semi-officielle, œuvre de son ami Marchamont Needham; d'autre part, il semble évident que Milton désapprouvait la politique reprise par le Bradshaw Press Act (20 septembre 1649) car, ayant à répondre de la publication du Catéchisme racovien, manifeste empreint de socinianisme, Milton déclare n'avoir fait que suivre ses convictions les plus profondes. ${ }^{9}$

En attendant, avec Areopagitica, il veut que le Parlement abroge le Licensing Order qu'il a promulgué dix-huit mois plus tôt (14 juin 1643): "by judging over again that Order which ye have ordain'd to regulate Printing" (2: 490-91). Cette loi "An Ordinance for the Regulating of Printing" (797-99) stipule effectivement: "no Book, Pamphlet, or paper... shall from henceforth be printed, bound, stitched or put to sale by any person(s) whatsoever, unlesse the same be first approved of and licenced under the hands of such person(s) as both, or either House of Parliament shall appoint." L'objectif visé était de supprimer "the great late abuses and frequent disorders in Printing many false forged, scandalous, seditious, libellous, and unlicensed Papers, Pamphlets, and Books to the

7. Frank Allan Patterson, The Student's Milton (New York: Crofts, 1930) xviii. Voir "For which uprightnesse and incorrupt refusall of what ye were incens'd to, Lords and Commons . . . I have yet a store of gratitude laid up" (Tetrachordon 2: 579).

8. En revanche, il serait normal, conseille-t-il à Cromwell, que l'auteur, qu'il confond avec l'imprimeur-éditeur, publiât ses découvertes "à [ses] risques et périls" (Pro populo anglicano defensio secunda 4: 679). S'il abuse de sa liberté d'expression, il s'expose aux sanctions prévues par elle, car "Impunity and remissenes, for certain are the bane of a Commonwealth" (Areopagitica 2: 527).

9. Voir William Riley Parker, Milton: A Biography (1968; Oxford: Clarendon, 1996) 395. 
great defamation of Religion and government." On publie, six jours plus tard, une liste de vingt-sept agents [trente au plus] chargés de remplir une tâche aussi ambitieuse. Tout nouvel ouvrage, avant d'être mis sous presse, devra encore avoir été légalement autorisé par un juré (warden) de la Corporation londonienne des libraires, laquelle jouissait alors d'un privilège exclusif; il sera inscrit sur les registres dudit corps de métiers, avec mention du nom de l'imprimeur. En outre, des perquisitions seront prévues pour découvrir les presses clandestines, ainsi que les ateliers où sont imprimés des écrits "délictueux," qu'ils soient simplement non autorisés ou qu'ils appartiennent à la Corporation ou à l'un de ses membres à part entière (freeman). Le matériel illicite sera mis hors d'usage, les livres prohibés saisis; les contrevenants, ainsi que quiconque se sera opposé à l'inspection, seront poursuivis en justice.

Après avoir mis en cause les Prélats dans le problème de l'éducation ("the Universities... have been poyson'd and choak'd under your governance [Animadversions 1: 718]), plaidé pour l'abrogation de "this papal and unjust restriction of divorce" (Tetrachordon 2: 706) et milité pour l'abolition de la pyramide épiscopale, le pamphlétaire, avec Areopagitica, œuvre pour la suppression de la censure - "the removal of an undeserved thraldom upon lerning" (539). Selon Milton, l'autorisation préalable empêche les Anglais de faire avancer la réforme de l'Église, condition sine qua non d'un retour à la paix civile. ${ }^{10}$

Il sera question, dans une première partie, d'étudier l'argumentaire: l'Écriture au service des écrits. Milton lui fera subir quelques réajustements. Une seconde section traitera des implications théologico-politiques venant appuyer la position miltonienne. Enfin, un chapitre de conclusion viendra redéfinir le livre.

Il est un principe sur lequel les protestants ne pouvaient que s'accorder: l'autorité exclusive de la Bible - "its absolute sufficiency, and supremacy inviolable," déclare Milton dans Of Reformation (1: 570). En effet, n'est-elle pas seule dépositaire de la Parole de Dieu, synonyme de salut pour quiconque l'écoute? Aussi est-elle le Livre par excellence au XVIIe siècle. Christopher Hill, dans l'introduction de The English Bible and the Seventeenth-Century Revolution, résume bien la situation avec

10. Milton, Reason of Church-Governement 1: 798 ("the corrupted estate of the Church is both the cause of tumult, and civill warres"). 
un poème daté de 1640: "It is the Book of God. What if I should / Say, god of books."11

Cette autorité exclusive de l'Écriture ne s'accommode guère des réflexions humaines consignées dans des livres relégués au rang de vanités. Et il ne faut pas oublier que quelques semaines plus tôt, Milton, à l'instigation de Samuel Hartlib, exposait sa vision de l'éducation, où la connaissance s'avérait essentiellement livresque. Dans $A d$ patrem, il remercie son père de lui avoir permis de s'adonner à l'étude. C'est que Milton est profondément humaniste: par l'étude des auteurs de l'Antiquité, l'homme est en mesure d'apprendre la vertu, de remonter jusqu'à Dieu: "The end . . . of learning is to repair the ruins of our first parents by regaining to know God aright, and out of that knowledge to love him, to imitate him, to be like him, as we may the neerest by possessing our souls of true vertu" (Areopagitica 2: 366-67).

Il en va différemment dans Areopagitica, où les livres évoqués par le polémiste sont plus que variés, avec une mention particulière pour les ouvrages de l'Antiquité, qu'ils nient, comme Protagoras, l'existence de Dieu, pour embrasser la philosophie épicurienne (2: 494-95) [que Milton réduit injustement à la seule recherche du plaisir] ou qu'ils fassent une satire des gens, comme dans les poèmes de Lucilius ou les comédies d'Aristophane ou de Plaute.

L'Écriture est le livre le plus cité dans Areopagitica; elle joue un rôle prépondérant dans l'argumentation. Statistiquement, quarante-huit références scripturaires sont plus ou moins identifiables, soit plus d'une référence par page. Elles revêtent diverses formes, allant de la seule citation à la simple allusion, avec quelques développements allégoriques. Trente-deux références, soit deux sur trois, proviennent du Nouveau Testament dans la Version Autorisée. Leur agencement est significatif: Areopagitica s'ouvre avec un lieu commun: l'évocation de "la grande prostituée," que l'on identifie à l'Église catholique romaine (2: 506; Ap. 17.1-5), et se termine sur l'idée que l'on doit laisser faire quiconque prêche au nom de Jésus (2: 567; Luc 9.49-50).

Ces références bibliques s'articulent principalement autour de quatre grands axes:

Il s'agit d'abord de prouver la licité de la lecture de n'importe quel livre: le patriarche Moïse aurait été initié à la magie, aux sciences occultes égyptiennes; le prophète Daniel comprenait "la science," c'est-à-dire qu'il pouvait interpréter les songes; pour aider ses auditeurs païens à sortir de

11. Christopher Harvey, Complete Poems, ed. Alexander B. Grosart (London, 1874)21. 
l'ignorance, l'apôtre Paul s'efforce, dans sa prédication, de retrouver dans le paganisme des analogies avec son message, d'où l'insertion de plusieurs éléments profanes dans son discours. ${ }^{12}$ Après les exemples vient le principe: "Prove all things; hold fast that which is good" (1 Thess. 5.21), ce à quoi Milton voudrait ajouter l'injonction apocryphe dans Actes 10.13: "Rise Peter, kill and eat, leaving the choice to each mans discretion" (Areopagitica 2: 511-12). Ce faisant, il déplace le sens de l'extase de Pierre à Joppé: $s$ "'il ne faut déclarer immonde ou impur aucun homme" (Actes 10.28), s'il est permis au juif de fréquenter le païen sans risque de souillure contagieuse, dans Areopagitica il s'agit, par analogie, de la licence qu'a le lecteur de consommer n'importe quel livre, bon ou mauvais, assuré que leur "digestion" ne saurait le perturber. Les livres, nourriture de l'esprit, ne peuvent corrompre, "[unless] their mind and conscience is defiled" (Tit. 1.15). Et Milton de poursuivre: les interdits alimentaires ayant été abolis, ce n'est plus ce qui entre dans la bouche de l'homme qui le souille, mais ce qui en sort, "that which cometh out of the mouth," ce qui provient du cœur - comprenez: les atteintes qu'il porte à son prochain (Matt. 15.10-20). En langage miltonien, cela veut dire que l'homme a pleine latitude d'absorber mentalement ce qu'il veut. Dieu a pourvu, par la raison, à ce qu'il jouisse de la liberté de choix, de la proairesis aristotélicienne; Il lui a donné la raison afin qu'il exerce son jugement.

Ces deux derniers passages, remis dans leur contexte, auraient pu justifier la censure: en effet, l'Épître à Tite constate la présence de faux docteurs ("there are many unruly and vain talkers, especially they of the circumsion") avant de conclure que l'on doit les museler ("[their] mouths must be stopped"). "Those things which proceed out of the mouth come from the heart; and they defile the man," dit Matthieu (15.18): elles polluent. En conséquence, il faudrait les réprimer. Évidemment, Milton ne prend dans l'Écriture que les morceaux susceptibles de soutenir sa cause. C'est alors qu'il est en mesure de poser un nouveau principe: "God ... left arbitrary the dyeting and repasting of our minds; as wherein every mature man might have to exercise his owne leading capacity" (Areopagitica 2: 513). Car, réfute Milton, si la Bible informe que "l'excès de lecture" épuise le corps (Eccles. 12.12: "much study is a weariness of the flesh"), elle dit qu'il n'est que fatigant; elle ne proscrit la lecture d'aucun ouvrage (Areopagitica 2: 514). Et il ne convient pas de muer un appel à une prudente réserve, lequel consisterait à dire "Lisez avec

12. Citation d'Épiménide de Cnossos: "in him [God] we live, and move, and have our being" (Actes 17.28); d'Aratos: "we are also his offspring" (17.28); de Ménandre (1 Cor. 15.33). 
modération," en interdiction formelle - Je vous défends de lire tels livres. Cependant, Milton relève par avance une objection: si des Éphésiens en voie de conversion brûlèrent des livres en public (Actes 19.19), il l'explique parce qu'ils traitaient de magie ("Many of them also which used curious arts"), sens qu'il estime précisé par la version syriaque; mais surtout, il affirme qu'il ne faut y voir qu'un acte volontaire d'ordre privé, que leur démarche n'a aucune force obligatoire pour les futurs croyants (Areopagitica 2: 514).

Une deuxième série de références bibliques contribue à un développement sur la nature de l'homme. Milton délaisse la question des livres pour aborder le problème fondamental de la Connaissance. Dès lors qu'il considère les problèmes du mal ou de la vérité, il abandonne chemin faisant le problème de la censure pour prôner la liberté d'opinion. Ce glissement capital apparaît lorsque Milton en vient à évoquer l'humaine condition. Adam, ayant refusé d'observer une loi extérieure "the law which proceeded from the mouth of God; Gen. 2.17: do not eat of this"13 -, le péché, synonyme de malheur, de mort, est entré dans le monde. Sans que la Providence y soit pour rien, il a opacifié l'œil de sa raison. Conséquence: l'homme ne vit plus dans la félicité avec Dieu, "souverain bien," puisqu'il s'en est laissé séparer, qu'il a naufragé "in this world of evil" (Gal. 1.4). Son expérience lui permet désormais d'acquérir la connaissance du mal présent, du bien possible, c'est-à-dire qu'il ne connaît le bien qu'en fonction du mal, indirectement: "It was from out the rinde of one apple tasted, that the knowledge of good and evill as two twins cleaving together leapt forth into the World. And perhaps this is that doom which Adam fell into ... of knowing good by evill" (Areopagitica 2: 514). Il lui faut tenir compte de la réalité mauvaise, mais non afin de l'expérimenter, de faire le mal dans l'espoir qu'advienne le bien. Connaître Dieu, Milton le sait, consiste à garder Ses commandements, à obéir à "his reveled wil" (Doctrine \& Discipline of Divorce 2: 292). L'homme ne fera qu'étudier les diverses manifestations du mal, pour le réprouver, s'en dégluer, s'efforcer de faire son contraire, la vertu: "whatsoever things are true, honest, just, pure, lovely, [and] of good report" (Phil. 4. 8).

Il en découle deux conséquences: primo, l'épreuve s'avère indispensable pour l'exercice de la vertu - d'où l'utilisation miltonienne de l'image de l'athlète dans le stade (1 Cor. 9.24-25); secundo, nul ne saurait détenir la vérité ici-bas: Milton s'en prenait vigoureusement à

13. Voir Milton, De doctrina christiana 6: 382. 
ceux qu'il voyait comme des accapareurs, "an Oligarchy of twenty ingrossers" (Areopagitica 2: 558), assimilés pour l'occasion aux forges philistines vers lesquelles les Israélites devaient se rendre pour l'affûtage de leurs outils en fer (1 Sam. 13.19-20;2: 536). Le 20 juin 1643 paraissait un document officiel, où les livres avaient été classés en neuf catégories: (a) "Books of Divinity"; (b) "Law Books"; (c) "Physick and Chyrurgery Books"; (d) "Civil and Canon Law Books"; (e) "Books of Heraldry, Titles of Honour and Arms"; (f) "Books of Philosophy, History, Poetry, Morality and Arts"; (g) "Declarations, Ordinances, Fast-Sermons, and Other Things Agreed on by Order of One or Both Houses of Parliament"; (h) "Small Pamphlets, Portractures, Pictures, and the Like"; (i) "Mathematicks, Almanacks, and Prognostications."14 Vingt-et-une personnes furent nommément désignées de manière permanente pour procéder à l'examen préalable; elles se répartissaient comme suit: douze pour les traités de théologie, quatre pour les livres de droit, une pour l'héraldique, trois seulement pour les lettres et une pour la dernière catégorie.

Si Milton incluait le livre dans les plaisirs de l'homme, avec des récréations ou divertissements comme la musique, les danses, les chants ou, plus globalement, les rapports sociaux (Areopagitica 2: 523-26), il voyait l'objectif primordial de l'écriture dans la réforme de la société selon l'Écriture, dans la recherche de la vérité, qu'il estimait inscrite dans les livres, y compris profanes: "heathen Writers ... with whom is bound up the life of human learning" (518). La verité fait l'objet de nombreuses images: elle est fontaine jaillissante (Psaumes 85.11), denrée commerciale la plus précieuse (Matt. 13.45-46); vigoureuse (Éph. 4.14-15), elle est aussi vulnérable. Elle est sagesse, crainte du Seigneur (Prov. 2.4-5); ondoyante, elle est moins avenante que l'erreur (1 Rois 22.1-36; 2 Cor. 10.10), car l'homme ne voit pas, ne choisit pas comme Dieu le fait (1 Cor. 1.26-27).

Le troisième groupe de références se rapporte à l'Église. On quitte résolument le problème de "the prohibition of Printing" (Areopagitica 2: 548 ) pour parler de religion. Tolérance réciproque, unité, paix. Tel est le message des passages bibliques auxquels Milton se réfère. Un dernier pan de références concerne la liberté chrétienne, que nous développerons plus loin.

14. Voir Charles H. Firth, and Robert S. Rait, Acts and Ordinances of the Interregnum 1642-60, 3 vols. (London: Professional Books Ltd, 1982) 1: 186-87. 
Mis au service de la liberté des livres, le Livre avait également permis à Milton de donner une portée philosophique à son discours. Encore fallait-il pouvoir pleinement l'assumer dans ses implications théologico-politiques.

Si pour John Foxe, dans le Livre des martyrs, la découverte de l'imprimerie fut un bienfait de Dieu, la censure, selon Milton, est l'œuvre de Satan: issue du concile de Trente, elle serait plus précisément l'œuvre de l'Inquisition dominicaine espagnole. Son objectif est évident: prévenir l'avancée du protestantisme. Elle est l'instrument de la Contre-Réforme: "licencing was first establisht and put in practice by Antichristian malice and mystery on set purpose to extinguish ... the light of Reformation, and to settle falshood" (Areopagitica 2: 548). Un Index librorum prohibitorum fut publié en 1559 . Cependant, le polémiste récrit les livres d'histoire, car la censure émane de la prérogative royale; en effet, dès 1530 , une proclamation d'Henri VIII stipule: "no maner of person(s) take upon hym or them to printe any boke(s) in englische tong, concernynge holy scripture ... ontyll such tyme as the same boke(s) be examyned and approued by the ordinary of the diocese"; huit ans plus tard, le roi, voulant empêcher, en sus des erreurs, les opinions séditieuses, élargissait la censure à l'ensemble des écrits imprimés en anglais: l'Acte de Suprématie avait effacé la barrière séparant le non-conformisme de la subversion. Six membres de son Conseil privé ou "other such as his highness shall appoynte" remplaçaient les officiers cléricaux. ${ }^{15}$

Le décret de 1637 faisait suite à celui de 1586; par l'Ordonnance du 14 juin 1643, un noyau dur de Presbytériens à l'Assemblée de Westminster ravivait la censure, dans le but, selon Milton, d'affamer les esprits, en empêchant à quiconque d'imprimer ses pensées: "this project of licencing . . . hath caught some of our Presbyters" (Areopagitica 2: 493). À moins que le Parlement ne se soit laissé séduire par l'Humble Pétition de la Corporation des libraires (24 août 1643): ceux-ci auraient voulu "exercer une suprématie sur leurs prochains" (570), garder le monopole sur la profession ou encore assurer l'évasion des livres. rebelles, à savoir des brochures pro-royalistes. En fait, il s'agissait de préparer l'union politico-religieuse avec l'Écosse, le Solemn League and Covenant, ratifié le 25 septembre 1643, notamment son paragraphe 2 .

La censure s'oppose au plan rédempteur de Dieu, qui répand sa grâce en suscitant des livres. Si la profusion de francs écrits issus des presses

15. Voir Fredrick S. Siebert, Freedom of the Press in England 1476-1776 (Urbana: U of Illinois $P, 1952)$ 46-49. 
de Londres témoigne d'un enthousiasme réel, d'une énergie sublime, d'efforts opiniâtres, Dieu en est non seulement la fin - chacun s'agite pour apporter sa contribution à "the approaching Reformation" -, mais encore la source: "the earnest and zealous thirst after knowledge and understanding which God hath stirr'd up in this City" (Areopagitica 2: $559,554)$. Les moyens, Dieu nous les donne également, dès lors qu'Il suscite des hommes d'exception pour Le voir: "God . . raises to his own work men of rare abilities, and more then common industry." Et nul n'est en droit de s'enorgueillir, parce que Dieu se révèle à l'entendement: "what does he ... but reveal Himself to his servants" $(566,553)$. Les Anglais, depuis John Wyclif, sont les premiers à recevoir Sa grâce, mais ils n'ont absolument rien fait pour la mériter. ${ }^{16}$ "This Nation [was] chos'n before any other" par la seule volonté divine (552). Pour prôner la liberté de la presse, il est nécessaire de soutenir que l'homme dispose du libre arbitre. Milton en rend compte de deux manières:

Soit que le libre arbitre, ayant perdu son efficacité dans la Chute, violation de la loi divine, fût en partie restauré par la vocation. L'homme agit parce qu'il est agi, Dieu récompense Ses dons en lui: la liberté naturelle qu'Il lui procure l'autorise à élire ce que l'entendement aura discerné. Ainsi faut-il a priori museler la censure puisque l'homme saura éliminer les mauvais livres.

Soit que la cause du refus ou de l'acceptation de la grâce initiale réside dans les dispositions naturelles de l'homme, dans le souhait. Certains seront "ordonnés," bien disposés ou propres au royaume de Dieu (Luc 9.62). Alors, le libre arbitre serait un vestige de son état primitif: l'homme est suffisamment pourvu de raison innée pour s'orienter dans la vie concrète, résister de lui-même à ses penchants pervers. À lui de le vouloir. Cela nous amène à une seconde lecture d'Areopagitica: l'Angleterre a une propension à chercher la vérité ("a Nation . . . so prone to seek after knowledge" [2: 554]); ${ }^{17}$ elle est virtuellement capable de connaissance ("a Nation . . . of a quick, ingenious, and piercing spirit" [551]), sens rendu par le trope "such a towardly and pregnant soile" (554), avec une allusion à la parabole du semeur en Matt. 13.3-9. Et son Parlement en est "the immediate cause" (559). Ce sol prometteur, pour fructifier, faire "a knowing people," doit encore être fécondé par de "wise

16. "[T]he favour and the love of heav'n we have great argument to think in a peculiar manner propitious and propending towards us" (Areopagitica 2: 552). "[Yet] we mark not the method of his counsels, and are unworthy" (2: 553).

17. Voir Milton, Reason of Church-Governement 1: 796 ("the Englishman ... bears a naturall disposition of much reverence and awe towards the Deity"). 
and faithfull labourers" (554). Ce sont les auteurs des "good Books" (500); ils représentent un surplus de grâce, que Dieu, dans son appel, accorde aux Anglais en raison de leurs qualités. Il n'est pas question d'accepter ou de refuser librement ce qu'ils auront à nous dire, mais d'accueillir favorablement leur message. Un palier est franchi: au nom du pouvoir de poursuivre volontairement le bien, il est impératif d'admettre les écrits, du moins les "serious and elaborat writings" d'un "free and knowing spirit" (531).

S'ils jouissent du libre arbitre, les Anglais possèdent encore la liberté chrétienne: celle-ci se définit d'abord comme l'abrogation de la loi mosaïque, Christ ayant effacé par son sang sur la croix le document accusateur, le Décalogue inscrit sur des Tables de pierre - aussi, en raison de sa non-maniabilité, ne convenait-il pas de parler de livre. "That handwriting," dit la version autorisée de la Bible, désignait l'humanité coupable: il était synonyme de mort. Cette liberté propre au Chrétien renvoie ensuite à un éventail de choses indifférentes, ni bonnes ni mauvaises en soi: les livres en feraient partie. Enfin, elle est la charité fraternelle, car il s'agit d'édifier le temple de Dieu. Cette liberté chrétienne, que Milton devait également rendre politique, allait lui servir à préconiser la tolérance des sectes protestantes, des "moderate varieties and brotherly dissimilitudes" (2: 555) - celle des consciences délicates, problème que voudrait résoudre l'ordonnance du 13 septembre $1644 .^{18}$

À l'instar de William Dell, dans Trial of Spirits (1653), Milton pense que le croyant, temple rationnel de Dieu, est "the only book in which God now writes his New Testament." ${ }^{19}$ Autrement dit, sa conscience est "l'Écriture non écrite," lieu interne où Dieu parle à l'homme, où l'homme, par l'effet de l'Esprit régénérateur, écoute Dieu, dialogue avec Lui. Cette loi intérieure est supérieure à "l'Écriture externe" (De doctrina christiana 6: 587); du moins celle-ci aura-t-elle fait l'objet d'une intériorisation. Milton la relègue au second plan. Car il est d'avis que le Texte est corrompu, qu'il ne faut désormais se fier qu'à l'Esprit Saint: si la décence exige la suppression de quelques passages, ${ }^{20} \mathrm{l}^{\mathrm{E}} \mathrm{E}$ criture est parfois obscure:

18. "[A]u prix de sa propre servitude, il a assis [leur] liberté politique sur une base solide" (Pro populo anglicano defensio 4: 375). Voir 1 Cor. 7.23.

19. Cité dans Stephen R. Honeygosky, Milton's House of God (Columbia: U of Missouri P, 1993) 224.

20. Milton, Areopagitica 2: 517 ("[It] oftimes relates blasphemy not nicely, it describes the carnal sense of wicked men not unelegantly, it brings in holiest men passionately murmuring against divine providence"). 
"the Bible ... in other great disputes answers dubiously and darkly to the common reader" (Areopagitica 2: 517). En outre, il semble que Milton n'envisage plus la Bible que dans sa dimension littéraire: dans Reason of Church-Governement, Milton écrit que l'Apocalypse a les allures d'une grande tragédie (1: 815); Apollinaire de Laodicée, rapporte le pamphlétaire dans Areopagitica, a essayé de verser des sujets bibliques dans le genre théâtral, la poésie, le dialogue socratique, jusqu'à en adapter une grammaire (Areopagitica 2: 509). "[T]hat best of books," que Milton célébrait dans An Apology against a Pamphlet (1: 933), "within whose sacred context all wisdome is infolded" (Animadversions 1: 747), a profondément évolué. Tout comme les livres issus de l'intelligence humaine: en fait, les livres que la raison condamne ne sont ni "temptations" (Areopagitica 2: 521), causes de chute ou de rechute, ni "vanities" (521), perte de temps, mais "working mineralls" (521). Ils en viennent presque à se substituer au Livre, si l'on considère leur fin, car ils servent à découvrir, à réfuter, à prévenir ou à éclairer $(512-13) .{ }^{21}$

Si le livre est de nouveau réifié dans l'expression "such a universall thing as books are," il s'ouvre aussitôt à une redéfinition surprenante: "what ever we hear or see, sitting, walking, travelling, or conversing may be fitly call'd our book" (Areopagitica 2: 527, 528). En définitive, le livre désigne l'expérience humaine, les relations de l'individu avec son environnement. Il ne renvoie plus à un contenant (l'objet; "the whole ware-house of his religion [544]), ni à un contenu (le sujet; "our richest Marchandize, Truth" [548]), mais il signifie la réaction de l'homme dans ses rapports au monde. Le livre devient plus largement l'éducation qu'il se fait à lui-même. Toutes nos pensées trouvent leur origine dans la sensation, mouvement causé en l'homme par l'action des objets extérieurs, écrit Thomas Hobbes dans Leviathan. ${ }^{22}$ L'éducation miltonienne fait apparaître que l'on progresse d'une connaissance du monde extérieur vers

21. Comparez 2 Tim. 3.16.

22. Thomas Hobbes, Leviathan, 1651, trans. François Tricaud (Paris: Sirey, 1971) 12. Sur l'importance de l'expérience, Milton rejoint Hobbes, bien que leurs philosophies restent distinctes. Pour Hobbes comme pour Milton, il y a deux espèces de connaissance, mais leur nature diffère. Hobbes distingue, d'une part, "la connaissance du fait" (Leviathan 79), d'autre part, "la connaissance des consécutions, de la dépendance d'un fait à l'égard de l'autre" (43). $\grave{\Lambda}$ l'approche scientifique de la connaissance, Milton substitue une vision morale. "[T] hat [which] rests in the contemplation of naturall causes and dimensions" définit pour le polémiste "lower wisdom," tandis que la perception de "any thing distinctly of God, and of his true worship, and what is infallibly good and happy in the state of mans life, what in it selfe evil and miserable, though vulgarly not so esteem'd" constitue "the only high valuable wisdom indeed" (Reason of Church-Governement 1: 801). 
celle de soi, être social, pour parvenir au langage, où l'homme, zôon politikon, réagit constamment par rapport à son environnement. La méthode que préconise Milton part logiquement des choses sensibles, de "the visible and inferior creature," pour pénétrer dans les réalités abstraites ou remonter jusqu'à Dieu (Of Education 2: 369). L'idée sera reprise dans Paradise Lost: "In contemplation of created things / By steps we may ascend to God" (5.511-12).

S'il incite à l'action, qu'il s'agisse de chercher son salut ou de promouvoir le bien public, le livre en fournit également le moyen puisque l'homme acquiert sa liberté par l'éducation. Celle-ci est proprement philosophique. Traduction du "zeal for wisdom" (Artis logica 8: 212), elle se définit comme l'apprentissage de la vertu morale.

Plus encore, le livre est action - exercice de la liberté d'agir; les livres ou les faits ne sont plus dépositaires de la pensée ou des stimuli de leur auteur, mais ils dépendent de la participation de chacun. Milton ne parle pas d'opinions, mais d'actions: "actions which issue out of [a man]" (Areopagitica 2: 513). Aussi dépasse-t-il le cadre de la liberté d'imprimer, "the Liberty of unlicenc'd Printing" (486), et celui de la liberté d'expression. S'il s'exclame: "Give me the liberty to know, to utter, and to argue freely according to conscience" (560), s'il demande la parrèsia, liberté de langage, il revendique davantage que la liberté de parole: il veut pouvoir agir sans contrainte, selon ce qu'il aura détcrminé en sa conscience. Cette liberté doit s'exercer spécialement en matière d'ecclésialité - dans les livres de controverse religieuse, parce que l'heure s'y prête (541). S'il ne plaide pas pour la liberté des mœurs, il s'agit pour chacun de vivre sa vie comme il l'entend, pourvu qu'il soit en accord avec sa raison - seule censeur légitime. L'État devra promulguer moins de lois pour laisser place à la seule persuasion (527). À Dieu d'inscrire sur Son livre de vie les hommes bons.

Dans An Apology against a Pamphlet, Milton identifiait le véritable écrivain à un vrai poème, "a composition, and patterne of the best and honourablest things" (1: 890). ${ }^{23}$ Son objectif: louer les nobles actions de ses compatriotes. C'est ce que Milton fait dans Areopagitica, œuvre en prose dans laquelle il déploie sa veine poétique. S'il le définit comme "a Homily" (2: 491) où il plaide pour la liberté d'écrire ou de lire n'importe

23. Le prosateur voulait devenir "an interpreter \& relater of the best and sagest things among mine own Citizens throughout this Illand in the mother dialect" (Reason of ChurchGovernement 1: 811-12). 
quel ouvrage ("freedom of writing" [541]; "freedom of learning" [541]), son discours va bien au-delà. Il est, en lui-même, le lieu de la célébration, un hymne à la gloire de la liberté des Anglais: "this whole Discourse propos'd will be a certaine testimony, if not a Trophey [of our] Countries liberty" (487), parce qu'il leur est donné de discuter de choses que nul n'a jamais abordées (557). Tout cela parce qu'ils disposent d'un libre arbitre, malgré l'omnipotence de Dieu. En fait, leur liberté est en Dieu, causalité immanente de leur agir: "We cannot be given the gift of will unless we are also given freedom of action, for that is what free will means," écrit Milton dans De doctrina christiana (6: 457), lequel, en raison des idées hétérodoxes qu'il développe, ne fut jamais publié du vivant de l'auteur. ${ }^{24}$ Et au moment où Milton demandait pour la première fois "a more free permission of printing" pour les sectes, ${ }^{25}$ le nombre de pamphlets était passé de 22 en 1640 à 1966 deux ans plus tard, relève Vincent Blasi dans un article récent. ${ }^{26}$ S'il était à l'évidence consacré au problème de la liberté de la presse, Areopagitica en venait doublement à faire la presse de la liberté.

\author{
Christophe TOURNU \\ Université Pierre Mendès-France - Grenoble 2
}

24. Le manuscrit fut découvert à Whitehall en 1823 par Robert Lemon Sr. (17791835), conservateur des documents officiels de Sa Majesté. Charles Richard Sumner (1790-1874), évêque de Winchester, le publia dans sa version originale et en proposa une traduction (Cambridge, 1825). Récemment, une polémique a surgi au sujet de la paternité du De doctrina christiana, avec le professeur William Bridges Hunter notamment. Voir William B. Hunter, "Visitation Unimplor'd": Milton and the Authorship of De doctrina christiana (Pittsburgh: Duquesne UP, 1998).

25. Milton, An Apology against a Pamphlet (1642): "[That] a more free permission of printing at some times might be profitable, in such a question especially wherein the Magistrates are not fully resolv'd" (1: 907).

26. Vincent Blasi, "Milton's Areopagitica and the Modern First Amendment," Yale Law School Occasional Papers 1 (1995). Cet article est actuellement disponible sur, Internet à l'URL suivante:

$<$ http://www.yale.edu/lawweb/lawschool/occpaper/blasi.htm> 